\title{
Relationship Between Gut Microbial Health, Exercise, Race, and Hypertension: Updating Mechanistic Perspective
}

\author{
Marc D Cook $^{1 *}$, Mesha Guinyard ${ }^{2}$, Lanna Anderson ${ }^{2}$, Austin Robinson ${ }^{3}$ and TJ Exford ${ }^{1}$ \\ ${ }^{1}$ North Carolina Agriculture and Technology State University, Department of Kinesiology, USA \\ ${ }^{2}$ North Carolina Agriculture and Technology State University, Department of Biology, USA \\ ${ }^{3}$ Auburn University, School of Kinesiology, USA
}

*Corresponding author: Marc D Cook, North Carolina Agriculture and Technology State University, Greensboro, NC Address: 1601 E. Market St. Greensboro, NC 27411 USA.

Received Date: August 11, 2019

Published Date: August 21, 2019

\begin{abstract}
The gut microbiome (the sum of microbes that populate the intestines) has recently been shown to have an active role in the development and promotion of disease. Hypertension, the number one and most modifiable cardiovascular disease risk factor, has been associated with a distinct gut microbial profile of reduced short- chain fatty acid production. Strategies to improve hypertension have been well-studied. Less appreciated is that exercise can improve gut microbial characteristics. Therefore, the impact of exercise on hypertension may include specific exercise-mediated improvements of gut short-chain fatty acid production, but the relationship between exercise-induced gut short-chain fatty acid production and reductions in blood pressure has not been characterized. Even more understudied is the phenomenon of the significant burden of hypertension in African Americans and the physiological factors (e.g. gut microbiome) that participate in this racial health disparity. This short communication will provide a contextual update concerning the mechanistic perspective of exercise induced improvement in hypertension and potential impact of exercise in mediating gut health and this significant racial health disparity.
\end{abstract}

Keywords: Hypertension; Short-chain fatty acids; Exercise; African americans

\section{Introduction}

The symbiotic bacteria in our gut perform multiple beneficial tasks including, but not limited to, maturing our immune system, breaking down our food and extract nutrients, processing fibers in our diet to produce beneficial products called short-chain fatty acids (SCFA), and defending against pathogenic microbes [1]. Yang et al. [2] reported that hypertension was associated with a low abundance of SCFA producing gut microbes in humans and rodents. Following this study, and a subsequent report by Li et al. [3] concerning the relationship between gut dysbiosis and hypertension (HTN), it has become evident that gut microbes participate in the HTN status of an individual. Further, targeted treatment of gut microbial health should be included in interventions to reduce blood pressure (BP). One of the most potent prevention and treatment strategies for cardiovascular diseases (CVD), specifically HTN, is chronic moderate to vigorous exercise [4]. We have recently shown that chronic exercise improves gut microbial characteristics in animal and human models of exercise training [5,6]. Exercisemediated alterations in gut microbial characteristics likely play a role in exercise-mediated reductions in BP, but this association is significantly understudied.

\section{Hypertension (HTN)}

Although HTN is a US public health concern affecting people of all racial/ethnic backgrounds, it is now estimated that HTN effects over 55\% African Americans (AA) while only $42 \%$ of Caucasians (CA) are afflicted [7]. Bolin et al. [8] recently reported that of AA receiving pharmacological treatment for $\mathrm{HTN}$, only 58\% reached their ideal BP, while $65 \%$ of their medicated CA counterparts achieved controlled BP. Even when comparing AAs to CA with BP below conventional HTN thresholds, there is still a disproportionate amount of sub-clinical manifestations of BP-related target organ 
injury in AA [9]. The pathogenesis of HTN within the AA community makes it difficult to treat due to its intertwined multifactorial involvement with salt sensitivity, renin angiotensin system, vascular response, and obesity [9]. Not only can untreated HTN lead to heart disease and stroke, but other factors that contribute to early mortality and morbidity in AA include environmental characteristics and behavioral factors [8]. Interestingly, genetic programming [10], dietary habits [11,12], environmental stressors [13], and sedentary behavior (e.g., no exercise) all have a role in the promotion of gut dysbiosis and poorer cardiovascular health [14].

\section{Gut Microbiome}

It is unclear whether gut dysbiosis contributes to the pathogenesis of HTN or is an unrelated comorbid condition, but research has shown clear associations with HTN and a distinct gut microbial profile. Previous studies have investigated the association of gut microbial characteristics and BP when comparing non- hypertensive subjects, pre-hypertensive (pHTN) subjects, and HTN subjects [3]. Gut microbial characteristics consisted of reduced microbial abundance, variation, and consistency with a greater Firmicutes/Bacteroidetes ratio. Control subjects exhibited a more diverse and rich gut flora comprising of Faecalibacterium, Oscillibacter, Roseburia, Bifidobacterium, Coprococcus, and Butyrivibri. Meanwhile the pHTN and HTN subjects had an underrepresentation of the bacterium present in healthy controls and had an overrepresentation of Prevotella and Klebsiella. Lastly, performing a human fecal microbial transplant (FMT) into germ free mice further demonstrated the role the HTN gut microbial profile in elevating BP. More evidence supporting the effects of FMT were shown when salt-sensitive rats were transplanted with salt-resistant rat microbiota, resulting in a higher BP in the recipient [3]. Metabolic health improvements were also shown to be transferrable via FMT from exercise trained and diet-controlled mice into obese mice [15]. These data implicate the gut microbiota have a relationship with its host that dictates health and disease risk.

\section{Exercise and Short-Chain Fatty Acids}

Diet and exercise have been linked to controlling factors associated with HTN (e.g., body mass, lipid and blood glucose profiles). Regular participation in exercise has been shown to significantly reduce all-cause morbidity and mortality from CVD [16]. The benefits of regular exercise are not limited to the heart, but include: protecting blood vessels against accelerated atherosclerosis, regeneration of vascular and muscle tissue, improved muscle function, and attenuating age-related muscle loss, improving systemic immune function, and improving our gut health by increasing SCFA producing microbes and improving our gut barrier function [16].

Our group and others have reported that diet and exercise are associated with beneficial changes of the gut microbiota in animals andhumans $[5,6,15,17]$.Laietal.[15] demonstrated exercise-induced stress impacts gut microbiota via several mechanisms including the release of hormones such as corticotropin, noradrenaline, and serotonin through the activation of the hypothalamic-pituitaryadrenal axis. These changes promote the expansion of microbes with proficiency in energy production through glycolysis and oxidative phosphorylation. It is now understood that gut microbes interact with the brain via stimulation of the enteric nervous system resulting in the production of neurotransmitters such as serotonin, melatonin, and acetylcholine [18].

SCFA have been shown to mediate BP via actions reviewed by Pluznick [19]. Briefly, SCFA can stimulate vasorelaxation of blood vessels via SCFA receptors in the vascular endothelium to reduce BP [19]. SCFA have also been shown to regulate vascular tone and lower BP via its interactions with renin production in the kidney [20]. In humans, we recently we reported that just six weeks of aerobic exercise training in healthy young lean and obese individuals increased SCFA microbes and SCFA production capacity of butyrate, propionate, and acetate [6]. We also found that the gut microbiome exhibits a "use it or lose it profile" post exercise training. After a 6-week washout period of no exercise, the beneficial gut microbial profile reverted to pre-exercise profile.

Recent evidence suggests that the gut microbial profile may be related to exercise capacity. The overgrowth of opportunistic pathogenic bacteria such as Candida, Yersinia, Salmonella, and Shigella species has been found in older patient population who have significantly reduced exercise capacity due to hypertension and subsequent chronic heart failure [21]. Further, a recent study by Scheiman et al. [22] reported that the microbial species Veillonella atypica was elevated in elite marathon runners and, when isolated from the fecal microbiome of the runners and given to mice, elicited a significant increase in exercise endurance. The proposed mechanism was that these lactate fermenting microbes metabolized the lactate into the SCFA propionate to potentially reduce exercise-induced lactate burden and provided energy for sustained exercise performance. It is well known that chronic aerobic exercise is important for reducing BP and may be related to exercise-induced changes in gut microbial SCFA production to improve sustained vascular compliance and health.

\section{Conclusion}

The interactions between gut microbial health, exercise, and HTN are evident. Growing evidence demonstrates that gut microbial health is essential to BP status. As AA have the greatest risk of HTN in the US, treatment strategies need to consider targeting gut microbial health, especially considering low gut SCFA production has been associated with HTN. An understudied mechanism of action of exercise on CV health may be related to exercises' capacity to balance gut microbial characteristics, improve gut SCFA production, and subsequently lower BP.

\section{Acknowledgements}

Publication of this review is supported by American Heart Association (AHA) Career Development Award (CDA) 18CDA34110444 awarded to Marc D. Cook. 


\section{Conflict of Interest}

All authors declare there are no competing or conflicting interests.

\section{References}

1. Valdes AM, Walter J, Segal E (2018) Role of the gut microbiota in nutrition and health. BMJ 361: k2179.

2. Yang T, Santisteban MM, Rodriguez V, Li E, Ahmari N, et al. (2015) Gut dysbiosis is linked to hypertension. Hypertension 65(6): 1331-140.

3. Li J, Zhao F, Wang Y, Chen J, Tao J, et al. (2017) Gut microbiota dysbiosis contributes to the development of hypertension. Microbiome 5(1): 14.

4. Börjesson M, Onerup A, Lundqvist S, Dahlöf B (2016) Physical activity and exercise lower blood pressure in individuals with hypertension: narrative review of 27 RCTs. Br J Sports Med 50(6): 356-361.

5. Cook MD, Allen JM, Pence BD, Wallig MA, Gaskins HR, et al. (2016) Exercise and gut immune function: evidence of alterations in colon immune cell homeostasis and microbiome characteristics with exercise training. Immunol Cell Biol 94(2): 158-163.

6. Allen JM, Mailing LJ, Niemiro GM, Moore R, Cook MD, et al. (2018) Exercise Alters Gut Microbiota Composition and Function in Lean and Obese Humans. Med Sci Sports Exerc 50(4): 747-757.

7. Benjamin EJ, Muntner P, Alonso A, Bittencourt MS, Callaway CW, et al. (2019) Heart Disease and Stroke Statistics-2019 Update: A Report from the American Heart Association. Circulation 139(10): e56-e528.

8. Bolin LP, Crane PB, Powell JR, Horne CE, Floegel TA (2018) Factors associated with physical activity in African Americans with hypertension Appl Nurs Res 41: 62-67.

9. Ortega LM, Sedki E, Nayer A (2015) Hypertension in the African American population: A succinct look at its epidemiology, pathogenesis, and therapy. Nefrologia 35(2): 139-145.

10. Goodrich JK, Waters JL, Poole AC, Sutter JL, Koren O, et al. (2014) Human genetics shape the gut microbiome. Cell 159(4): 789-799.

11. Ou J, Carbonero F, Zoetendal EG, DeLany JP, Wang M, et al. (2013) Diet, microbiota, and microbial metabolites in colon cancer risk in rural Africans and African Americans. Am J Clin Nutr 98(1): 111-120.
12. O'Keefe SJ (2016) Diet, microorganisms and their metabolites, and colon cancer. Nat Rev Gastroenterol Hepatol 13(12): 691-706.

13. Bailey MT (2016) Psychological Stress, Immunity, and the Effects on Indigenous Microflora. Adv Exp Med Biol 874: 225-246.

14. Serino M, Blasco-Baque V, Nicolas S, Burcelin R (2014) Far from the eyes, close to the heart: dysbiosis of gut microbiota and cardiovascular consequences. Curr Cardiol Rep 16(11): 540.

15. Lai ZL, Tseng CH, Ho HJ, Cheung CKY, Lin JY, et al. (2018) Fecal microbiota transplantation confers beneficial metabolic effects of diet and exercise on diet-induced obese mice. Sci Rep 8(1): 15625.

16. Fiuza-Luces C, Santos-Lozano A, Joyner M, Carrera-Bastos P6, Picazo 0 , et al. (2018) Exercise benefits in cardiovascular disease: beyond attenuation of traditional risk factors. Nat Rev Cardiol 15(12): 731-743.

17. Allen JM, Berg Miller ME, Pence BD, Whitlock K, Nehra V, et al. (1985) Voluntary and forced exercise differentially alters the gut microbiome in C57BL/6J mice. J Appl Physiol 118(8): 1059-1066.

18. Carabotti M, Scirocco A, Maselli MA, Severi C (2015) The gut-brain axis: interactions between enteric microbiota, central and enteric nervous systems. Ann Gastroenterol 28(2): 203-209.

19. Pluznick JL (2017) Microbial Short-Chain Fatty Acids and Blood Pressure Regulation. Curr Hypertens Rep 19(4): 25.

20. Pluznick JL, Protzko RJ, Gevorgyan H, Peterlin Z, Sipos A, et al. (2013) Olfactory receptor responding to gut microbiota-derived signals plays a role in renin secretion and blood pressure regulation. Proc Natl Acad Sci U S A 110(11): 4410-4415.

21. Yu Y, Mao G, Wang J, Zhu L, Lv X, et al. (2018) Gut dysbiosis is associated with the reduced exercise capacity of elderly patients with hypertension. Hypertens Res 41(12): 1036-1044.

22. Scheiman J, Luber JM, Chavkin TA, MacDonald T, Tung A. et al. (2019) Meta-omics analysis of elite athletes identifies a performance-enhancing microbe that functions via lactate metabolism. Nat Med 25(7): 11041109. 